\title{
Turbulence dissipation rate derivation for meandering occurrences in a stable planetary boundary layer
}

\author{
G. A. Degrazia ${ }^{1}$, A. Goulart ${ }^{2}$, J. Costa Carvalho $^{3}$, C. R. P. Szinvelski ${ }^{1}$, L. Buligon ${ }^{1}$, and A. Ucker Timm ${ }^{1}$ \\ ${ }^{1}$ Departamento de Física, Universidade Federal de Santa Maria, Santa Maria, RS, Brazil \\ ${ }^{2}$ Centro de Tecnologia de Alegrete, UNIPAMPA/UFSM, Alegrete, RS, Brazil \\ ${ }^{3}$ Faculdade de Meteorologia, Universidade Federal de Pelotas, Pelotas, RS, Brazil
}

Received: 3 September 2007 - Published in Atmos. Chem. Phys. Discuss.: 26 October 2007

Revised: 18 February 2008 - Accepted: 18 February 2008 - Published: 25 March 2008

\begin{abstract}
A new formulation for the turbulence dissipation rate $\varepsilon$ occurring in meandering conditions has been presented. The derivation consists of a MacLaurin series expansion of a lateral dispersion parameter that represents cases in which turbulence and oscillatory movements associated to the meandering events coexist. The new formulation presents the identical physical premises contained in the classical and largely used one, but the new formulation derived from meandering situations is expressed in terms of the loop parameter $m$ that controls the absolute value of the negative lobe in the meandering autocorrelation function. Therefore, the $m$ magnitude regulates the turbulence dissipation rate. This dissipation rate decreases for cases in which turbulence and low frequency horizontal wind oscillations coexist and increases for a fully developed turbulence. Furthermore, a statistical comparison to observed concentration data shows that the alternative relation for the turbulent dissipation rate occurring in situations of meandering enhanced dispersion is suitable for applications in Lagrangian Stochastic dispersion models.
\end{abstract}

\section{Introduction}

Lagrangian turbulent velocity correlation coefficient is an important physical quantity in turbulent diffusion problems. Generally, the use of this autocorrelation coefficient in the Taylor statistical diffusion theory allows calculating the dispersion parameters associated to the turbulent diffusion modeling studies in the Planetary Boundary Layer (PBL) (Taylor, 1921).

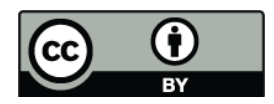

Correspondence to: G. A. Degrazia (degrazia@ccne.ufsm.br)
For a stationary and isotropic turbulence, the two-time Lagrangian velocity correlation coefficient may be written as

$\rho_{L_{i}}(\tau)=\frac{\overline{u_{i}^{\prime}(t) u_{i}^{\prime}(t+\tau)}}{\overline{u_{i}^{\prime 2}}}$,

where $\tau$ is the time lag, $u_{i}^{\prime}(t)$ is the Lagrangian turbulent velocity of a fluid particle at time $t, i=1,2$ and $3 ; u_{i}^{\prime}$ represents the turbulent wind components in $x, y$ and $z$ directions $\left(u_{1}^{\prime}=u^{\prime}, u_{2}^{\prime}=v^{\prime}\right.$ and $\left.u_{3}^{\prime}=w^{\prime}\right)$.

Based on the Taylor statistical diffusion theory, the following formula has been proposed by Frenkiel (1953) to represent the turbulent Lagrangian autocorrelation coefficients

$\rho_{L_{i}}(\tau)=\exp \left(-\frac{\tau}{\left(m^{2}+1\right) T_{L_{i}}}\right) \cos \left(\frac{m \tau}{\left(m^{2}+1\right) T_{L_{i}}}\right)$.

This functional form is composed of the product of the classical exponential function (representing the autocorrelation function for a fully developed turbulence) by the cosine function (describes the meandering phenomenon associated to the observed low frequency horizontal wind oscillations). This latter allows Eq. (2) to reproduce the negative lobes observed in the meandering autocorrelation functions (Anfossi et al., 2005). The Frenkiel function is a hybrid formula described in terms of $T_{L i}$, the Lagrangian integral time scale for a fully developed turbulence, and $m$, the loop parameter, which controls the meandering oscillation frequency associated to the horizontal wind. Indeed, the $m$ parameter controls the negative lobe absolute value in the autocorrelation function and hence establishes the meandering phenomenon magnitude (Anfossi et al., 2005).

Recently, Oettl et al. (2005) and Goulart et al. (2007) proposed a theory on meandering atmospheric flow in low

Published by Copernicus Publications on behalf of the European Geosciences Union. 
wind speed situations. This theory shows that, in simplified conditions, the forecast equation for mean wind in the PBL (Stull, 1988) provides a particular solution that is capable of describing the meandering behavior. Here, we succinctly present this derivation employing the three-dimensional Reynolds-average momentum conservation equation in an atmospheric boundary layer (Holton, 1992). Neglecting viscosity terms, this equation that describes the horizontal mean wind may be written as

$$
\frac{\partial \overline{u_{i}}}{\partial t}+\overline{u_{j}} \frac{\partial \overline{u_{i}}}{\partial x_{j}}=-\delta_{i 3} g+f_{c} \varepsilon_{i j 3} \overline{u_{j}}-\frac{1}{\bar{\rho}} \frac{\partial \overline{p_{i}}}{\partial x_{i}}-\frac{\partial \overline{\left(u_{i}^{\prime} u_{j}^{\prime}\right)}}{\partial x_{j}}(3)
$$

where $i, j=1,2$ and $3 ; \overline{u_{i}}$ represents the mean wind components in $x, y$ and $z$ directions $\left(\overline{u_{1}}=\bar{u}, \overline{u_{2}}=\bar{v}\right.$ and $\left.\overline{u_{3}}=\bar{w}\right)$ averaged over a certain time interval, $f_{c}$ is the Coriolis parameter, $\varepsilon_{i j 3}$ is the Levi-Civita tensor, $\bar{\rho}$ is the mean density, $\overline{p_{i}}$ is the mean pressure and $g$ is the gravity acceleration.

The complexity of Eq. (3) does not allow it to be analytically solved, except under some appropriate simplifying assumption yields from Eq. (3) an analytical solution. However, we assume that all the horizontal and vertical gradients of the horizontal wind velocity components and pressure may be considered as constant. Furthermore, the $\bar{w}$ magnitude, which characterizes subsidence in fair weather boundary layer conditions, may range from 0 to $0.1 \mathrm{~ms}^{-1}$ (Stull, 1988). Thus, assuming these values in Eq. (3), the terms containing $\bar{w}$ and horizontal gradients of $\bar{w}$ could be neglected. With these simplifications and assuming the hydrostatic balance condition yields

$$
\left\{\begin{array}{l}
\frac{\partial \bar{u}}{\partial t}=-a_{1} \bar{u}+b_{1} \bar{v}+c_{1} \\
\frac{\partial \bar{v}}{\partial t}=-a_{2} v+b_{2} \bar{u}+c_{2}
\end{array},\right.
$$

where $\quad a_{1}=\frac{\partial \bar{u}}{\partial x}, \quad a_{2}=\frac{\partial \bar{v}}{\partial y}, \quad b_{1}=f_{c}-\frac{\partial \bar{u}}{\partial y}, \quad b_{2}=-f_{c}-\frac{\partial \bar{v}}{\partial x}$, $c_{1}=-\frac{1}{\bar{\rho}} \frac{\partial \bar{p}}{\partial x}-\left[\frac{\partial \overline{\left(u^{\prime} u^{\prime}\right)}}{\partial x}+\frac{\partial \overline{\left(u^{\prime} v^{\prime}\right)}}{\partial y}+\frac{\partial \overline{\left(u^{\prime} w^{\prime}\right)}}{\partial z}\right]$ and

$c_{2}=-\frac{1}{\bar{\rho}} \frac{\partial \bar{p}}{\partial y}-\left[\frac{\partial \overline{\left(u^{\prime} v^{\prime}\right)}}{\partial x}+\frac{\partial \overline{\left(v^{\prime} v^{\prime}\right)}}{\partial y}+\frac{\partial \overline{\left(v^{\prime} w^{\prime}\right)}}{\partial z}\right]$.

Considering the $a_{1} a_{2}, b_{1}, b_{2}, c_{1}$ and $c_{2}$ terms as constants, the system (4) becomes a first-order linear differential equation system, where the horizontal wind components in Eq. (4) may be written as a second-order linear ordinary differential equation with constant coefficients

$\frac{d^{2} \bar{u}}{d t^{2}}+B \frac{d \bar{u}}{d t}+C \bar{u}=D$,

where $B=a_{1}+a_{2}, C=a_{1} a_{2}-b_{1} b_{2}$ and $D=a_{2} c_{1}+b_{1} c_{2}$.

According to the values of roots $r_{1}$ and $r_{2}$ from the auxiliary equation, there are three cases in the solution of Eq. (5). We select the case that presents oscillatory behavior, which means the condition $B^{2}-4 C<0$, in the auxiliary (or characteristic) equation for the given differential Eq. (5), $r_{1,2}=\frac{B \pm \sqrt{B^{2}-4 C}}{2}$.
Therefore, the following solution for $\bar{u}(t)$ and $\bar{v}(t)$ is obtained

$\bar{u}(t)=e^{-p t}\left[\alpha_{1} \cos (q t)+\alpha_{2} \sin (q t)\right]+\frac{D}{C}$

and

$$
\begin{aligned}
\bar{v}(t) & =e^{-p t} \\
& {\left[\begin{array}{l}
\left.\frac{-\alpha_{1} p+\alpha_{2} q+a_{1} \alpha_{1}}{b_{1}} \cos (q t)+\frac{-\alpha_{2} p-\alpha_{1} q+a_{1} \alpha_{2}}{b_{1}} \sin (q t)+\right] \\
+\frac{D}{C} \frac{a_{1}}{b_{1}}-\frac{c_{1}}{b_{1}}
\end{array}\right] }
\end{aligned}
$$

with

$p=\frac{B}{2}=\frac{1}{2}\left(\frac{\partial \bar{u}}{\partial x}+\frac{\partial \bar{v}}{\partial y}\right)=0$ (nondivergent wind field),

$q=\frac{\sqrt{-B^{2}+4 C}}{2}, \alpha_{1}=u_{0}-\frac{D}{C}$,

$\alpha_{2}=\frac{1}{q}\left[v_{0} b_{1}-a_{1} u_{0}+c_{1}\right], \overline{u_{0}}=\bar{u}\left(t_{0}\right)$ and $\overline{v_{0}}=\bar{v}\left(t_{0}\right)$.

If the horizontal and vertical gradients of the turbulent momentum fluxes may be disregarded, a scale analysis allows the derivation of the following simplified form for Eqs. (6a) and (6b) (Oettl et al., 2005; Goulart et al., 2007).

$\bar{u}(t)=\alpha_{1} \cos (q t)$

and

$\bar{v}(t)=-\alpha_{1} \sin (q t)$,

The prototype wind field given by Eqs. (7a) and (7b) is a sound solution, namely a two-dimensional nondivergent wind field uniform on the horizontal plane. Therefore, these solutions describe the horizontal wind meandering behavior.

Expressing Eqs. (7a) and (7b) in an analytical functional form as

$U(t)=\alpha_{1} e^{-i q t}$,

and employing Eq. (8) into Eq. (1), the following turbulent velocity correlation coefficient is obtained

$\rho_{L u, v}=\cos (q \tau)-i \sin (q \tau)$,

In fact the correlation functions are different for the $u$ and $v$ components, and are dependent on the initial time, because the field is deterministic and unsteady. Only if an average over a finite number of periods (or over a long time) is taken, the correlation function turns out to be the same for both the components, and written as

$\rho_{L u, v}(\tau)=\cos (q \tau)$,

which presents the cosine functional form as in the Frenkiel autocorrelation function (Eq. 2). Anfossi et al. (2005) employed Frenkiel classical mathematical expression to reproduce autocorrelation functions observed during meandering periods, which presented negative lobes. 
Therefore, based on observational evidences (Anfossi et al., 2005) and on the fact that the oscillatory character, associated to the meandering phenomenon, described by the autocorrelation coefficient proposed by Frenkiel is derived from the first principles (forecast equation for mean wind), we assume in this study that the Frenkiel autocorrelation function captures well the physical properties of a fully developed turbulence as well as hybrid flow cases, in which turbulence and meandering occurrences coexist. Indeed, the Frenkiel formulation (Eq. 2) presents an empirical flexibility that allows represent observations in the PBL (Manomaiphiboon and Russel, 2003, and Anfossi et al., 2005). This imparts to Eq. (2) a heuristic validity.

The turbulence dissipation rate is a quantity frequently used in turbulent parameterizations applied to Lagrangian stochatic dispersion models. Indeed, formulations for the turbulence dissipation rate are extensively employed in turbulent diffusion models to simulate the transport of passive scalars and the dispersion of contaminants in a fully developed turbulence (Thomson, 1987; Luhar and Britter, 1989; Sawford, 1991; Wilson and Sawford, 1996; Yeung, 2002; Degrazia et al., 2005). In this study, employing the Frenkiel autocorrelation coefficient, we derive a new relation to the turbulence dissipation rate for distinct types of turbulent flows. This new relation presents the same physical contents as those classical derived by Hinze (1975) and Tennekes (1982), however the dissipation rate could be described not only for a fully developed turbulence, but also for situations in which the turbulence weakness allows the occurrence of the meandering phenomenon. With the above statements becomes evident the existence of a strong constraint between the turbulence dissipation rate and the Lagrangian stochastic dispersion models. Therefore to investigate the influence of this new dissipation rate in turbulent diffusion problems, it is introduced in a Lagrangian stochastic dispersion model in order to simulate diffusion experiments performed under low wind speed meandering conditions.

\section{Theoretical development}

The lateral dispersion parameter $\sigma_{y}$ is a statistical quantity fundamental for the dispersion modeling and for the derivation of turbulence dissipation rate functional form (Tennekes, 1982; Degrazia et al., 2005). From the Taylor statistical diffusion theory, this important parameter may be described as

$\sigma_{y}^{2}(t)=2 \sigma_{v}^{2} \int_{0}^{t}(t-\tau) \rho_{v}(\tau) d \tau$,

where $t$ is the travel time of the fluid particle and $\sigma_{v}$ is the standard deviation of the turbulent lateral velocity. The substitution of the Frenkiel autocorrelation function into Eq. (11) yields

$\sigma_{y}^{2}(t)=2 \sigma_{v}^{2} T_{L_{V}}\left\{t+\left(m^{2}-1\right) T_{L_{V}}-T_{L_{V}}\right.$

$$
\begin{aligned}
& \exp \left(\frac{-t}{\left(1+m^{2}\right) T_{L_{V}}}\right)\left[\left(m^{2}-1\right) \cos \left(\frac{m t}{\left(1+m^{2}\right) T_{L_{V}}}\right)\right. \\
& \left.\left.+2 m \sin \left(\frac{m t}{\left(1+m^{2}\right) T_{L_{V}}}\right)\right]\right\}
\end{aligned}
$$

It is well known that atmospheric dispersion in low wind speed conditions is mainly governed by meandering. In such conditions, differently than a diffusion generated by a fully developed turbulence, the airborne contaminants are dispersed over distinct angular sectors. Therefore, this oscillatory behavior is the physical mechanism that reduces the lateral turbulent diffusion of a plume. Thusly, as can be seen in Fig. 11 from Anfossi et al. (2005), Taylor's equation employing Frenkiel autocorrelation function (Eq. 12) captures well the role of meandering in altering the turbulent diffusion. For the special case $m=0$, the Eq. (12) reproduces the classical expression for the lateral dispersion parameter valid to a fully developed turbulence, which is obtained by substituting the classical exponential autocorrelation function $e^{-\tau / T_{L_{v}}}$ into Eq. (11) (Pope, 2000). Thus, the Eq. (12) represents a more general formulation since it describes hybrid cases, in which turbulence and oscillatory movements associated to the meandering occurrences coexist (weak turbulence conditions).

For $t<T_{L_{v}}$, a MacLaurin series expansion of the general dispersion parameter (Eq. 12) may be obtained

$$
\begin{aligned}
\sigma_{y}^{2}(t) & =2 \sigma_{v}^{2}\left[\frac{t^{2}}{2}-\frac{t^{3}}{6\left(1+m^{2}\right) T_{L v}}+\ldots\right] \\
& =\sigma_{v}^{2} t^{2}-\frac{\sigma_{v}^{2} t^{3}}{3\left(1+m^{2}\right) T_{L v}}+\ldots
\end{aligned}
$$

The comparison of Eq. (13) with the Taylor theorem (Eq. 11) for small diffusion times shows that the negative term in the right side of Eq. (13) contributes for the decrease on the hybrid dispersion parameter. From the physical point of view, representing the Taylor model in the spectral form (Degrazia et al., 2005) may be seen that this negative contribution results in the suppression of a number of degrees of freedom of the turbulent field associated to the high-frequency harmonics. As a consequence, it is reasonable to relate the term $\frac{\sigma_{v}^{2} t^{3}}{3\left(1+m^{2}\right) T_{L v}}$ to the inertial subrange high frequency eddies. This relationship was firstly obtained by Tennekes (1982) through the use of the Lagrangian structure function, the Lagrangian autocorrelation function and the inertial subrange Lagrangian turbulent spectrum. The Tenneke's development establishes the following formulation for the Lagrangian autocorrelation function in terms of inertial subrange quantities:

$\rho_{L_{v}}=1-\frac{C_{o} \varepsilon \tau}{2 \sigma_{v}^{2}}$

Employing relation (14) in the Taylor's theorem results in $\sigma_{y}^{2}(t)=\sigma_{v}^{2} t^{2}-\frac{C_{0} \varepsilon}{6} t^{3}$, which after comparison with the 


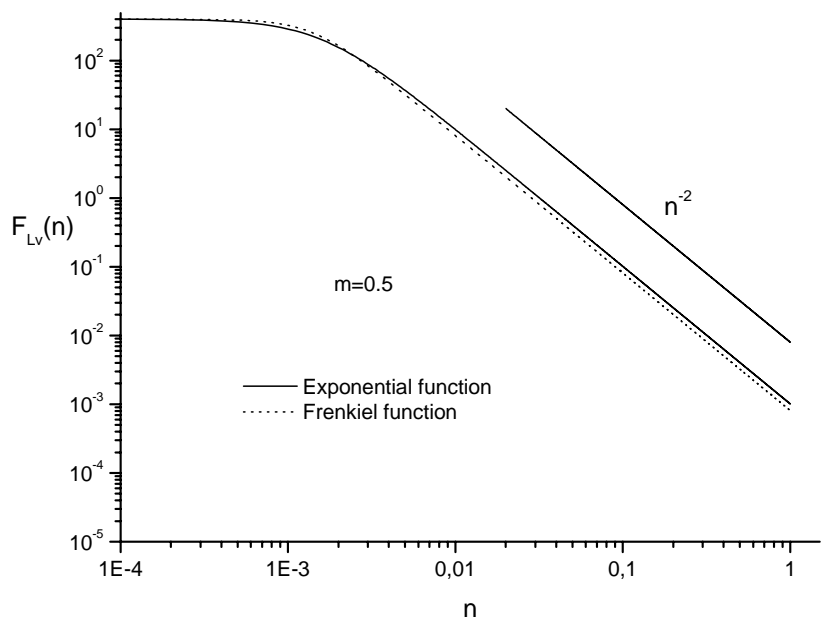

Fig. 1. Lagrangian turbulent energy spectrum in the inertial subrange, as determined from Eq. (2) for $m=0.5$.

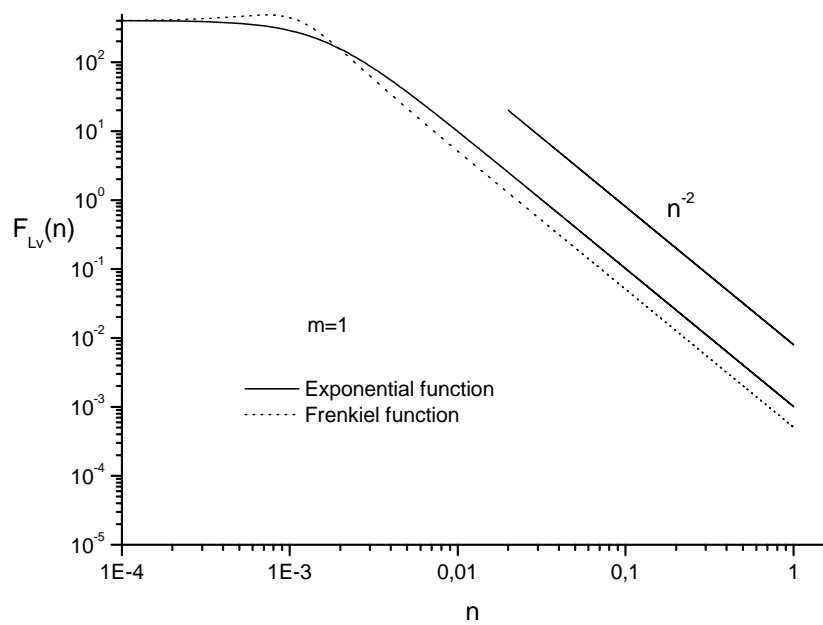

Fig. 2. Lagrangian turbulent energy spectrum in the inertial subrange, as determined from Eq. (2) for $m=1$.

truncated hybrid dispersion parameter (13), leads to the following fundamental relation

$\varepsilon=\frac{2}{\left(1+m^{2}\right) C_{0}} \frac{\sigma_{v}^{2}}{T_{L_{v}}}$

As seen from Eq. (15), this new formula for the turbulence dissipation rate preserves the fundamental physical premise that turbulence is dissipated at a rate proportional to the energy available and inversely proportional to the time scale of the energy containing eddies (Tennekes, 1982). However, as a new element in the relation (15), the parameter $m$ appears, which establishes a new formulation for the turbulent dissipation rate applied to hybrid situations, including the presence of low-frequency horizontal wind oscillations (meandering). In comparison with the classical turbulent dissipation rate derived from the exponential autocorrelation

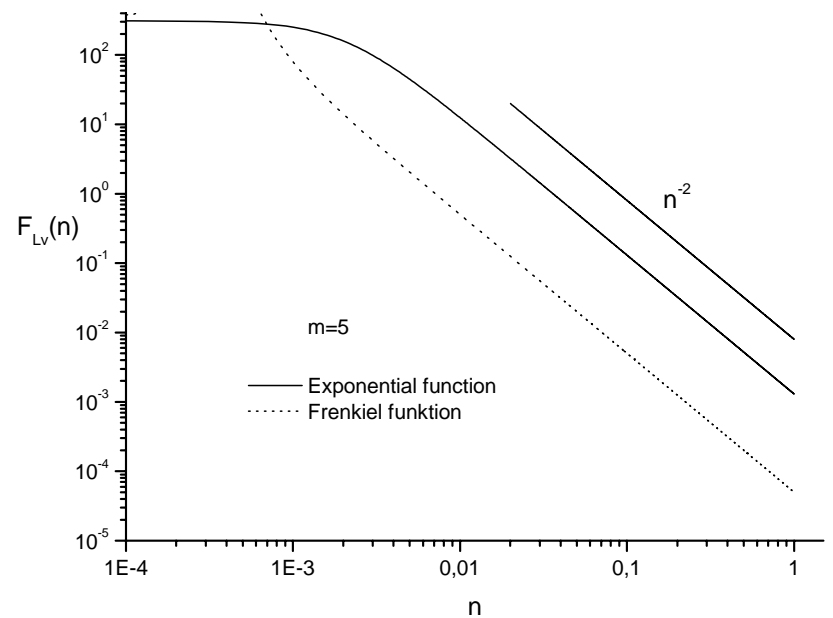

Fig. 3. Lagrangian turbulent energy spectrum in the inertial subrange, as determined from Eq. (2) for $m=5$.

function for a fully developed turbulence, the $m$ magnitude in the denominator controls the turbulence dissipation rate that will be larger for the fully developed turbulence case (small $\mathrm{m}$ ) and smaller for the meandering case (large $\mathrm{m}$ ). Therefore, we expect that the new functional form, Eq. (15), when employed in Lagrangian stochastic particle models, reproduces the dispersion process occurring under different types of turbulent flows in a more robust manner. Recently, Manomaiphiboon and Russel (2003), through consideration of mathematical and physical requirements, evaluated Eq. (2) for the special case $m=1$. They concluded that for this particular $m$ value, the Eq. (2) is appropriate to be used in turbulence studies because it complies well with most requirements. According to this procedure, we can see that the more general autocorrelation form, as given by Eq. (2), satisfies the condition $\int_{0}^{\infty} \rho_{L_{v}}(t) d t=T_{L_{v}}$ so that the Frenkiel formula is valid as long as $T_{L_{v}}$ is well defined. From the physical point of view, Eq. (2) satisfies the inertial subrange conditions as proposed by Kolmogorov (1941, K41 theory) and suggested by Tennekes (1979); Lagrangian velocity fluctuations should be in accordance with $F_{L_{v}}(n) \approx n^{-2}$ and $\rho_{L_{v}}(t)=1-c t$ where $c$ is a constant and $F_{L_{v}}(n)$ is the Lagrangian lateral turbulent spectrum. This is shown in Figs. 1, 2 , and 3 where a comparison between the energy spectra derived from both models (classical exponential autocorrelation function and Eq. 2) is presented. From these figures, we observe that the Frenkiel's formulation for distinct $m$ values (including $m$ values associated to strong negative lobes) captures the $n^{-2}$ falloff. Therefore, Eq. (2) agrees with the K41 theory. Furthermore, it can be also seen from Figs. 1, 2, and 3 that as $m$ increases, passing from non-meandering to meandering conditions, the turbulent energy associated to the inertial subrange frequencies (scales) decreases abruptly. Figure 4 exhibits an autocorrelation function characterized by a large negative lobe, which is associated to the meandering 
phenomenon. The minimum value of this negative lobe, and the general form in Fig. 4 agrees well with the meandering autocorrelation functions observed by Anfossi et al. (2005). Therefore, for large $m$ values characterizing the dominant presence of the meandering phenomenon in comparison with the fully developed turbulence, the turbulent energy of the inertial subrange becomes negligible and the low frequency horizontal wind oscillations (meandering) represent the fundamental energy source for the velocity variance observed in low wind speed conditions. At this point, it is relevant to consider that in the derivation of the Eq. (15), the most important result of the present study, we employed Eq. (12) for $t<T_{L_{v}}$. As the meandering period is greater than $T_{L_{v}}$, our analysis applies to conditions in which $t \ll T_{m}$, where $T_{m} \approx 2000 \mathrm{~s}$ is the average value of the meandering period (Anfossi et al., 2005). Therefore, a quasi stationary condition is guaranteed in the derivation of Eq. (15).

From the analysis above, we conclude that the Frenkiel hybrid formula (Eq. 2), represents a general expression that could be used to reproduce autocorrelation functions observed in the PBL. As a consequence, the turbulence dissipation rate derived from Frenkiel autocorrelation function and given by Eq. (15) represents a general functional form that converges to the classical relation $\varepsilon=\frac{2}{C_{0}} \frac{\sigma_{v}^{2}}{T_{L_{v}}}$ (obtained from the exponential autocorrelation function for a fully developed turbulence) when meandering effects are excluded (i.e. by setting $m \cong 0$ in Eq. 2).

\section{Test of the derived parameterization with the INEL tracer experiment}

The purpose of this section is to test our parameterization as given by Eq. (15) in a practical application and to show how it works. Therefore, a Lagrangian stochastic dispersion model using the turbulent dissipation rate containing the loop parameter $m$ (Eq. 15) has been employed to simulate the measured concentration data.

The concentration data employed in the comparison with the model were obtained from the low wind speed experiment performed in a stable boundary layer from the series of field observations conducted at the Idaho National Laboratory - INEL (Sagendorf and Dickson, 1974).

\subsection{Model description}

\subsubsection{Horizontal components}

Recently, Carvalho and Vilhena (2005) suggested an approach to obtain the solution of the Langevin equation for low-wind dispersion. The solution consists of the linearization of the Langevin equation as stochastic differential equation:

$\frac{d u_{i}}{d t}+f(t) u_{i}=g(t)$,

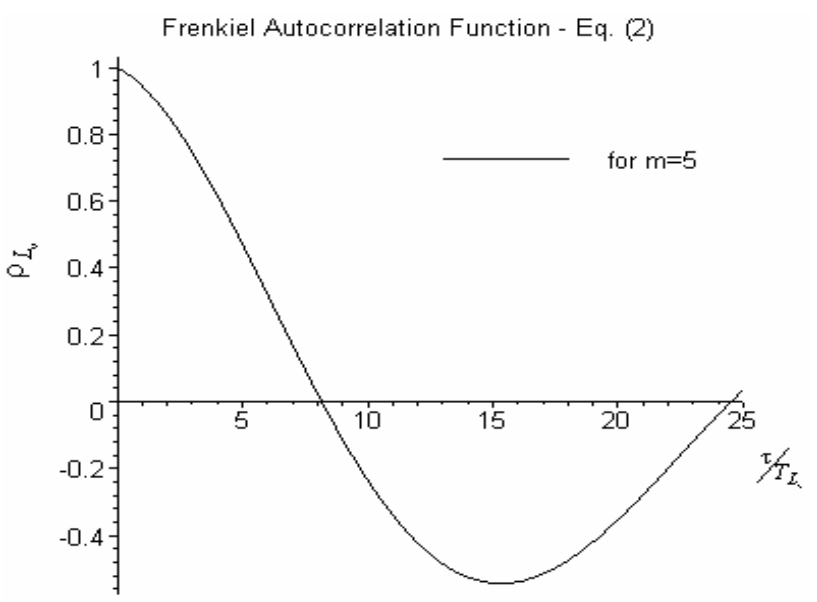

Fig. 4. Lagrangian autocorrelation function as given in Eq. (2) for $m=5$.

which has the well-known solution determined by the integrating factor $e^{t} f\left(t^{\prime}\right) d t^{\prime}$ :

$u_{i}=\frac{1}{\int^{t} f\left(t^{\prime}\right) d t^{\prime}} \int_{t_{0}}^{t} g\left(t^{\prime}\right) e^{t_{0}} f\left(t^{\prime}\right) d t^{\prime} d t^{\prime}$.

In order to embody the low wind speed condition in the Langevin equation, it is assumed that the function $f(t)$ is a complex function. Therefore, the exponentials appearing in Eq. (17) may be rewritten as:

$$
e^{t^{t} f\left(t^{\prime}\right) d t^{\prime}}=e^{t} p d t^{\prime}+\int_{t_{0}}^{t} i q d t^{\prime}
$$

or

$$
e^{\int_{0}^{t} f\left(t^{\prime}\right) d t^{\prime}}=e^{p t+i q t}
$$

Applying the Euler formula and neglecting the imaginary component because the wind speed is a real function, Eq. (17) becomes:

$u_{i}=e^{-p t} \cos (q t) \int_{t_{0}}^{t} g\left(t^{\prime}\right)\left(\frac{1}{e^{-p t^{\prime}} \cos \left(q t^{\prime}\right)}\right) d t^{\prime}$.

In Eq. (19), the term $e^{-p t} \cos (q t)$ is analogous to the autocorrelation function suggested by Frenkiel (1953) and written in a different way by Murgatroyd (1969), where $p$ and $q$ are given by $p=\frac{1}{\left(m^{2}+1\right) T}$ and $q=\frac{m}{\left(m^{2}+1\right) T}$ and $T$ is the time scale for a fully developed turbulence.

Using the Lagrangian particle models, the turbulence is considered as Gaussian in the horizontal directions $(i=1,2)$ 
and, therefore, Eq. (19) may be written as:

$$
\begin{array}{r}
u_{i}=e^{-p t} \cos (q t) \int_{t_{0}}^{t}\left(\frac{1}{e^{-p t^{\prime}} \cos \left(q t^{\prime}\right)}\right) \\
{\left[\beta_{i}+\gamma_{i} u_{i}^{2}+\left(C_{0} \varepsilon\right)^{1 / 2} \xi_{i}\left(t^{\prime}\right)\right] d t^{\prime}}
\end{array}
$$

where $\beta_{i}=\frac{1}{2} \frac{\partial \sigma_{i}^{2}}{\partial x_{j}}$ and $\gamma_{i}=\frac{1}{2 \sigma_{i}^{2}}\left(\frac{\partial \sigma_{i}^{2}}{\partial x_{j}}\right)$.

The Picard Method is applied to Eq. (20), assuming that the initial guess is determined from a Gaussian distribution. The generic iterative step is written as:

$$
\begin{aligned}
u_{i}^{n+1}= & e^{-p t} \cos (q t)\left\{u_{i}^{n}+\int_{t_{0}}^{t}\left(\frac{1}{e^{-p t^{\prime}} \cos \left(q t^{\prime}\right)}\right)\right. \\
& {\left.\left[\beta_{i}+\gamma_{i}\left(u_{i}^{2}\right)^{n}+\left(C_{0} \varepsilon\right)^{1 / 2} \xi_{i}\left(t^{\prime}\right)\right] d t^{\prime}\right\} . }
\end{aligned}
$$

Concerning the $m$ values, it is important to note two distinct cases about Eq. (21). For $m>1$, the meandering phenomenon has an effect upon the dispersion process. On the other hand, for $m=0$, the meandering phenomenon is vanished and Eq. (21) is written in terms of the exponential autocorrelation function $\left(e^{-t / \tau_{l}}\right)$, which Lagrangian particle models are usually employed in windy conditions. Therefore, the approach (21) is capable of simulating the contaminant dispersion in the PBL in both cases, that is, when the contaminant plume evolution is governed by eddies associated to a fully developed turbulence and exhibits a "fanning" kind of behavior (typical of windy conditions) and when the plume evolution is governed by low frequency horizontal wind oscillations (meandering behavior associated to low wind conditions).

For applications, the values obtained for the parameters $m$ and $T$ are calculated by the empirical formulation suggested by Carvalho and Vilhena (2005): $m=\frac{8.5}{(1+U)^{2}}, T=\frac{m T_{*}}{2 \pi\left(m^{2}+1\right)}$ and $T_{*}=200 m+500$.

\subsubsection{Vertical component}

For the vertical component, the Langevin equation is solved by the approach as suggested by Carvalho et al. (2005). While for the horizontal directions the PDF is considered as Gaussian, for the vertical direction the PDF is assumed to be non-Gaussian (to deal with non-uniform turbulent conditions and/or convection). In both cases, a Gram-Charlier PDF, which is given by the series of Hermite polynomials, may be adopted (Anfossi et al., 1997; Ferrero and Anfossi, 1998).

The Gram-Charlier PDF truncated to the fourth order is given by the following expression (Kendall and Stuart, 1977):

$P\left(r_{i}\right)=\frac{e^{-\left(r_{i}^{2} / 2\right)}}{\sqrt{2 \pi}}\left[1+C_{3} H_{3}\left(r_{i}\right)+C_{4} H_{4}\left(r_{i}\right)\right]$ where $r_{i}=u_{i} / \sigma_{i}, \sigma_{i}$ is the turbulent velocity standard deviation, $H_{3}$ and $H_{4}$ are the Hermite polynomials and $C_{3}$ and $\mathrm{C}_{4}$ their coefficients. In the case of Gaussian turbulence, Eq. (22) becomes a normal distribution, considering $C_{3}$ and $C_{4}$ equal to zero. The third order Gram-Charlier PDF is obtained with $C_{4}=0$.

Applying the Eq. (22) in the Fokker-Planck equation (Rodean, 1996), the Langevin equation may be written as:

$\frac{d u_{i}}{d t}+\alpha_{i} u_{i}=\beta_{i}+\gamma_{i}+\left(C_{0} \varepsilon\right)^{1 / 2} \xi_{i}(t)$,

where $\quad \alpha_{i}=\frac{15 C_{4}+1}{h_{i}} \frac{C_{0} \varepsilon}{2 \sigma_{u_{i}}^{2}}, \quad \beta_{i}=\left[f_{i}+r_{i}\left(15 C_{4}+1\right)\right] \frac{1}{h_{i}} \frac{C_{0} \varepsilon}{2 \sigma_{u_{i}}} \quad$ and $\gamma_{i}=\sigma_{i} \frac{\partial \sigma_{i}}{\partial x_{j}} \frac{g_{i}}{h_{i}}$

$j$ may assume $1,2,3$ and $j \neq i$ and $f_{i}, g_{i}$ and $h_{i}$ are expressions written as:

$f_{i}=-3 C_{3}-r_{i}\left(15 C_{4}+1\right)+6 C_{3} r_{i}^{2}+10 C_{4} r_{i}^{3}-C_{3} r_{i}^{4}-C_{4} r_{i}^{5}(24 \mathrm{a})$

$g_{i}=1-C_{4}+r_{i}^{2}\left(1+C_{4}\right)-2 C_{3} r_{i}^{3}-5 C_{4} r_{i}^{4}+C_{3} r_{i}^{5}+C_{4} r_{i}^{6}$

$h_{i}=1+3 C_{4}-3 C_{3} r_{i}-6 C_{4} r_{i}^{2}+C_{3} r_{i}^{3}+C_{4} r_{i}^{4}$.

Multiplying the integrating factor $\left[\exp \left(\int_{t_{0}}^{t} \alpha_{i} d s\right)\right]$ by all terms in Eq. (23), the following integral equation is obtained:

$$
\begin{gathered}
u_{i}=\exp \left(-\int_{t_{0}}^{t} \alpha_{i} d s\right)\left\{\int_{t_{0}}^{t} \exp \left(\int_{t_{0}}^{t^{\prime}} \alpha_{i} d s\right)\right. \\
\left.\left[\beta_{i}+\gamma_{i}+\left(C_{0} \varepsilon\right)^{1 / 2} \xi_{i}\left(t^{\prime}\right)\right] d t^{\prime}\right\},
\end{gathered}
$$

The Picard Method is now applied to Eq. (25), assuming that the initial value for the turbulent velocity is a random value supplied by a Gaussian distribution: The generic iterative step is written as:

$$
\begin{aligned}
u_{i}^{n+1}= & \exp \left(-\int_{t_{0}}^{t} \alpha_{i}^{n} d s\right)\left\{u_{i}^{0}+\int_{t_{0}}^{t} \exp \left(\int_{t_{0}}^{t^{\prime}} \alpha_{i}^{n} d s\right)\right. \\
& {\left.\left[\beta_{i}^{n}+\gamma_{i}^{n}+\left(C_{0} \varepsilon\right)^{1 / 2} \xi_{i}^{n}\left(t^{\prime}\right)\right] d t^{\prime}\right\} . }
\end{aligned}
$$

\subsection{Dispersion simulation}

The results of the proposed model are compared with concentration data collected under stable conditions in low wind speeds over flat terrain at the Idaho National Engineering Laboratory (INEL). The results have been published in a US National Oceanic and Atmospheric Administration (NOAA) report (Sagendorf and Dickson, 1974).

For simulations, the turbulent flow is considered as inhomogeneous only in the vertical direction and the transport is performed by the longitudinal component of the mean wind velocity. The horizontal domain was determined according to sampler distances and the vertical domain was set as equal 
Table 1. Statistical evaluation of the model results.

\begin{tabular}{ccccc}
\hline NMSE & $R$ & FA2 & FB & FS \\
\hline 0.14 & 0.93 & 0.73 & 0.08 & -0.23 \\
\hline
\end{tabular}

to the observed PBL height. The time step was maintained constant and it was obtained according to the value of the Lagrangian decorrelation time scale $\left(\Delta t=\tau_{L} / c\right)$, where $\tau_{L}$ must be the smallest value between $\tau_{L_{u}}, \tau_{L_{v}}, \tau_{L_{w}}$ and $c$ is an empirical coefficient set as equal to 10 . For each simulation, the number of particles released was $10^{6}$ and the concentration was obtained by counting the number of particles in volumes with dimensions $5 \mathrm{~m} \times 5 \mathrm{~m}$ and $0.5 \mathrm{~m}$ in height. Values of $\sigma_{i}$ and $\tau_{L_{i}}$ were parameterized according to scheme developed by Degrazia et al. (1996). For the horizontal components (Eq. 21) the parameterization of $C_{0} \varepsilon$ has been calculated from the Eq. (15) employing $m$ values given by the empirical formulation suggested by Carvalho and Vilhena (2005). For the vertical component, the Eq. (15) is employed with $m=0$. The third moment of the vertical velocity component is assigned according to Chiba (1978) and the fourth moment is calculated based on method suggested by Anfossi et al. (1997). The integration method used to solve the integrals appearing in Eqs. (21) and (26) was the Romberg technique.

Due to the wind direction variability, a full $360^{\circ}$ sampling grid was implemented. Arcs were laid out with radii of 100, 200 and $400 \mathrm{~m}$ from the emission point. Samplers were placed at intervals of $6^{\circ}$ on each arc for a total of 180 sampling positions. The receptor height was $0.76 \mathrm{~m}$. The tracer $\mathrm{SF}_{6}$ was released at a height of $1.5 \mathrm{~m}$. The $1 \mathrm{~h}$ average concentrations were determined by means of an electron capture gas chromatography. Wind speeds measured at levels 2, 4, $8,16,32$ and $61 \mathrm{~m}$ were used to calculate the coefficient for the exponential wind vertical profile. According to Brusasca et al. (1992) and Sharan and Yadav (1998), the roughness length used was $z_{0}=0.005 \mathrm{~m}$. The Monin-Obukhov length $L$ and the friction velocity $u_{*}$ were not available for the INEL experiment but may be roughly approximated. Then, $L$ may be written from an empirical formulation suggested by Zannetti (1990) and the stable PBL height $h$ was determined according to expression suggested by Zilitinkevich (1972).

The model performance is shown in Table 1 and Fig. 5 . Table 1 shows the result of the statistical analysis performed with observed and predicted ground-level centerline concentration values according to Hanna's (1989) statistical indices. Figure 5 shows the scatter diagram between observed and predicted concentration. Observing the results obtained, we may promptly conclude that the model simulates quite well the experimental data in low wind stable conditions. The statistical analysis reveals that all indices are within acceptable ranges, with NMSE, FB and FS values relatively near to zero and $R$ and FA2 relatively near to 1 .

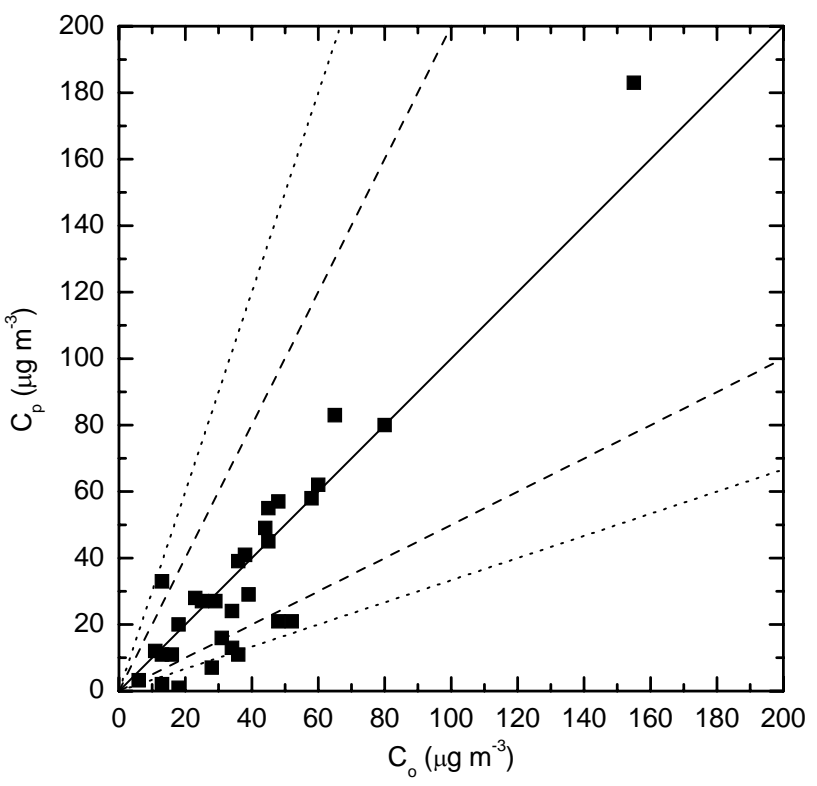

Fig. 5. Scatter diagram between observed $\left(C_{o}\right)$ and predicted $\left(C_{p}\right)$ ground-level centerline concentration values for the stable case. Dashed lines indicate factor of 2, dotted lines indicate factor of 3 and solid line indicates unbiased prediction.

\section{Conclusion}

In this study, a new formulation for the turbulence dissipation rate $\varepsilon$ has been derived and tested. Unlike the classical form, which is derived from the exponential autocorrelation function, this new expression (Eq. 15) is obtained from an autocorrelation function that describes negative lobes in the observed autocorrelation functions associated to the meandering phenomenon. Furthermore, the cosine function contained in this autocorrelation function, which describes the oscillatory behavior associated to the meandering events, has been derived from the forecast equation for mean wind in the PBL, when a number of simplifications are assumed. Therefore, the new formulation for the turbulence dissipation rate is expressed in terms of the loop parameter $m$ and may be employed to parameterize the turbulence dissipation rate associated to the low frequency horizontal wind oscillations (meandering phenomenon) occurring in low wind stable conditions. The new parameterization may be applied in situations in which turbulence and meandering phenomenon coexist. Consequently, the turbulence dissipation rate as given by Eq. (15) constitutes a general functional form that may be used in distinct turbulent flow patterns.

The presence of $m$ in Eq. (15) controls the turbulence dissipation rate. It will be larger for a well-developed turbulence (neglected $m$ value) and smaller for the physical state described by the turbulence and meandering phenomenon coexistence (hybrid situation). 
The present parameterization for the turbulence dissipation rate was evaluated and validated through the comparison with experimental data. Particularly, the results obtained by the Lagrangian stochastic dispersion model, employing the new formula for the turbulence dissipation rate, show that the model correctly represents the diffusion process in low wind speed stable conditions.

Considering the statistical evaluation and the scatter diagram given in Fig. 5, the new parameterization for the turbulence dissipation rate seemed to be suitable to simulate the meandering enhanced diffusion of passive scalars occurring in situations in which turbulence and meandering phenomenon coexist. Therefore, the main result of the present study is Eq. (15), which represents an alternative expression for the turbulence dissipation rate for hybrid flow cases and that has been derived from an autocorrelation function that reproduces experimental meandering data.

Acknowledgements. This work was partially supported by $\mathrm{CNPq}$ and CAPES.

Edited by: S. Galmarini

\section{References}

Anfossi, D., Ferrero, E., Sacchetti, D., and Trini Castelli, S.: Comparison among empirical probability density functions of the vertical velocity in the surface layer based on higher order correlations, Bound.-Lay. Meteorol., 82, 193-218, 1997.

Anfossi, D., Oettl, D., Degrazia, G. A., and Goulart, A.: An analysis of sonic anemomenter observations in low wind speed conditions, Bound.-Lay. Meteorol., 1(114), 179-203, 2005.

Brusasca, G., Tinarelli, G., and Anfossi, D.: Particle model simulation of diffusion in low wind speed stable conditions, Atmos. Environ., 26a, 707-723, 1992.

Carvalho, J. C., Nichimura, E. R., Vilhena, M. T. M. B., Moreira, D. M., and Degrazia, G. A.: An iterative langevin solution for contaminant dispersion simulation using the Gram-Charlier PDF, Environ. Mod. And Soft., 20(3), 285-289, 2005.

Carvalho, J. C. and Vilhena, M. T.: Pollutant dispersion simulation for low wind speed condition by the ils method, Atmos. Environ., 39, 6282-6288, 2005.

Chiba, O.: Stability dependence of the vertical wind velocity skewness in the atmospheric surface layer, J. Meteor. Soc. Japan, 56, 140-142, 1978.

Degrazia, G. A., Moraes, O. L. L., and Oliveira, A. P.: An Analytical method to evaluate mixing length scales for the planetary boundary layer, J. Appl. Meteor., 35, 974-977, 1996.

Degrazia, G. A., Acevedo, O. C., Carvalho, J. C., Goulart, A. G., Moraes, O. L. L., Campos Velho, H. F., and Moreira, D. M.: On the universality of the dissipation rate functional form and of autocorrelation function exponential form, Atmos. Environ., 39, 1917-1924, 2005.

Ferrero, E. and Anfossi, D.: Comparison of PDFs, closures schemes and turbulence parameterizations in Lagrangian Stochastic Models, Int. J. Environ. Pollut., 9, 384-410, 1998.
Frenkiel, F. N.: Turbulent diffusion: Mean concentration distribution in a flow field of homogeneous turbulence, Adv. Appl. Mech., 3, 61-107, 1953.

Goulart, A. G. O., Degrazia, G. A., Acevedo, O. C., and Anfossi, D.: Theoretical considerations of meandering winds in simplified conditions, Bound.-Lay. Meteorol., 125, 279-287, 2007.

Hanna, S. R.: Confidence limit for air quality models as estimated by bootstrap and jacknife resampling methods, Atmos. Environ., 23, 1385-1395, 1989.

Hinze, J. O.: Turbulence, MacGraw-Hill, New York, 790 pp, 1975.

Holton, J. R.: An Introduction to Dynamic Meteorology, Elsevier Academic Press, 535 pp., 2004.

Kendall, M. and Stuart, A.: The advanced theory of statistics, MacMillan, New York, 1977.

Kolmogorov, A. N.: The Local Structure of Turbulence in Incompressible Viscous Fluid for Very Large Reynolds Numbers, Dokl. Akad. Nauk. SSSR, 30, 301-305, 1941.

Luhar, A. K. and Britter, R. E.: A random walk model for dispersion in inhomogeneous turbulence in a convective boundary layer, Atmos. Environ., 9, 1911-1924, 1989.

Manomaiphiboon, K. and Russel, A. G.: Evaluation of some proposed forms of Lagrangian velocity correlation coefficient, Int. J. Heat Fluid Flow, 24, 109-712, 2003.

Murgatroyd, R. J.: Estimations from geostrophic trajectories of horizontal diffusivity in the mid-latitude troposphere and lower stratosphere, Q. J. Roy. Meteor. Soc., 95, 40-62, 1969.

Oettl, D., Goulart, A., Degrazia, G., and Anfossi, D.: A new hypothesis on meandering atmospheric flows in low wind speed condition, Atmos. Environ., 39, 1739-1748, 2005.

Pope, S. B.: Turbulent Flows, Cambridge University Press, Cambridge, UK, 2000.

Rodean, H. C.: Stochastic Lagrangian models of turbulent diffusion, American Meteorological Society, Boston, 84 pp, 1996.

Sagendorf, J. F. and Dickson, C. R.: Diffusion under low windspeed, inversion conditions, US National Oceanic and Atmospheric Administration Tech. Memorandum ERL ARL-52, 1974.

Sawford, B. L.: Reynolds number effects in Langragian stochastic models of turbulent dispersion, Phys. Fluids, A3, 1577-1586, 1991.

Sharan, M. and Yadav, A. K.: Simulation of experiments under light wind, stable conditions by a variable K-theory model, Atmos. Environ., 32, 3481-3492, 1998.

Stull, R. B.: An Introduction to Boundary Layer Meteorology, Kluver Academic Publishers, Dordrecht, Boston 666 pp, 1988.

Taylor, G. I.: Diffusion by continuous movements, Proc. London Math. Soc., 20, 196-211, 1921.

Tennekes, H.: The exponential Lagrangian correlation function and turbulent diffusion in the inertial subrange, Atmos. Environ., 12, 1565-1567, 1979.

Tennekes, H.: Similarity relation, scaling laws and spectral dynamics, in: Atmospheric Turbulence and Air Pollution Modeling, edited by: Nieuwstadt, F. T. M. and Van Dop, H., Reidel, Dordrecht, 37-68, 1982.

Thomson, D. J.: Criteria for the selection of stochastic models of particles trajectories in turbulent flows, J. Fluid Mech., 180, 611615, 1987.

Yeung, P. K.: Lagrangian investigation of turbulence, Ann. Rev. Fluid Mech., 34, 115-142, 2002.

Wilson, J. D. and Sawford, B. L.: Review of Lagrangian stochastic 
models for trajectories in the turbulent atmosphere, Bound.-Lay. Meteorol., 78, 191-210, 1996.

Zannetti, P.: Air Pollution Modeling. Computational Mechanics Publitions, Southampton, 444 pp, 1990.
Zilitinkevich, S. S.: On the determination of the height of the Ekman boundary layer, Bound.-Lay. Meteorol., 3, 141-145, 1972. 